\title{
Forgiveness as a Workplace Intervention: The Literature and a Proposed Framework
}

\author{
Susan R. Madsen \\ Janice Gygi \\ Scott C. Hammond \\ Utah Valley University \\ Suzanne F. Plowman \\ Brigham Young University
}

\begin{abstract}
The purpose of this review is to explore the literature related to interpersonal forgiveness in organizations and its possible implications for management and HRD theory and practice. It defines forgiveness and provides a theoretical framework for its consideration within the workplace environment. It also reviews and discusses the benefits and risks of forgiveness, the role of leadership in a forgiving culture, and the literature regarding related business interventions.
\end{abstract}

\section{Introduction}

Improving individual, team and organizational performance is a primary focus of management and human resource development (HRD). However, only in the past decade has the literature begun to report specific research on the effects of many of the psychological and behavioral constructs that influence performance and productivity of individual employees. In fact, organizational literature in management, organizational behavior, and other business fields has also done little to address many of these constructs. Cameron and Caza (2002) stated that only a few researchers (see citations later in paper for examples) have begun to "investigate dynamics in organizations that lead to the development of human strength, resiliency, and extraordinary performance" (p. 33) - with forgiveness being one such dynamic.

Within the management literature a few writers have argued that forgiveness is a construct that is important to address in the workplace environment. According to Aquino, Grover, Goldman, and Folger (2003), "Forgiveness should be an important concern of both organizational theorists and practicing managers because it is a way for individuals to repair damaged workplace relationships and overcome debilitating thoughts and emotions resulting from interpersonal injury" (p. 210). They argue: Humans working together have endless opportunities to offend or harm others, intentionally or unintentionally. An organization is a melee of relationships alternating between firm and sound, unconnected, sordid, broken and angry, and changing. The quality of healing broken and changing relationships should profoundly influence how well an organization functions as well as the nature of work life within organizations. (p. 214)

Bottom, Gibson, Daniels, and Murnigham (2002) declare effective relationship management, forgiveness being one important element, is critical to success in 
business. Kurzynski (1998) explained that "good working relationships are essential to organizational effectiveness" (p. 79). The bottom line is that, "other than the family, the workplace is the most powerful influence on the individual's life experience" (Butler \& Mullis, 2001, p. 260), yet the importance of forgiveness and its effect on work performance remains unexplored.

Cameron and Caza (2002) argued that forgiveness is one of the "relatively few universal human virtues" (p. 36) that all of the world's major religious traditions believe human beings should aspire to incorporate in their lives. Forgiveness is a construct that has its roots in religion. Some construct of forgiveness can be found in all of the world's major religions (Smith, 1991). But aside from being theological, every child in a sandbox learns the practical need for forgiveness as hurt feelings can escalate to eclipse any advantage of social interaction. We learn early in our lives to move on, to let go and be socially practical. Beyond the sandbox, the concept of forgiveness has the potential to be a factor in every major turning point in work life at the individual and organizational level. A termination, lay off, or reassignment can create a sustained individual hurt that may affect the rest of a career. A plant closing or organizational change that displaces or reassigns workers can create similar hurts. However, forgiveness remains "among the least understood virtues and one of the most difficult to attain" (Cameron \& Caza, 2002, p. 37). Hence, understanding the impact and effects of forgiveness (or lack of it) in the workplace is a complex undertaking.

\section{Purpose, Questions, and Design}

The purpose of this review is to explore the literature related to forgiveness, suggest a framework for its consideration in the workplace, and outline its possible implication for management and HRD theory and practice. The following questions were investigated: 1) What are definitions and theoretical frameworks of forgiveness in the literature? 2) What are the benefits and risks in practicing workplace forgiveness? 3) What are currently used and possible business interventions related to workplace forgiveness? and 4) How does this information contribute to new knowledge in management and HRD? This review is a content analysis of scholarly literature located in business, psychology, education, health, and general academic library databases found within most library systems. Most was found in the psychology and health indexes, but a few related articles were located in others. No articles were found in HRD literature, while only 12 were located in management. The keywords used for the search included forgive and forgiveness. Among the hundreds of articles located on the topic of forgiveness, the most applicable manuscripts to the specific focus of this paper were reviewed in-depth $(n=84)$. Because of length limitations, we used the articles that had the most relevant findings and could offer the most pertinent management and HRD implications.

\section{Definitions and Theoretical Framework}

Although many authors (e.g., Legaree, Turner, \& Lollis, 2007; Orr, Sprague, Goertzen, Cornock, \& Taylor, 2005) have admitted that there is no agreed-upon definition of 
forgiveness, exploring a number of definitions, theories, and models can provide insight into understanding the construct. Analyzing components of the various definitions of forgiveness provides the theoretical framework for this content analysis. In this review we discovered that nearly all definitions or frameworks acknowledged that forgiveness is not condoning, forgetting, or ignoring a hurtful action; however, as Enright and Coyle (1998) stated, "In genuine forgiveness, one who has suffered an unjust injury chooses to abandon his or her right to resentment and retaliation, and instead offers mercy to the offender" (p. 140). Scobie and Scobie (1998) concur with the elements of the previous definitions as they define forgiveness as

a conscious decision to set aside one's legitimate claim for retaliation or restitution for a damaging act committed by a significant other, in order for any, or all, of the following to occur: 1) the relationship, or a modified version of the relationship, to be restored; 2 ) the negative affects associated with the damaging act for the forgiver and/or the forgiven to be reduced; 3 ) the forgiver to cease playing the role of the victim, and the forgiven to be given the opportunity to make amends; and 4) the forgiver and the forgiven to gain release from the dominating effect of the damaging act. (p. 382)

They also noted that this decision is made without "condoning or minimizing the effects of the damaging act" (p. 382). McCullough, Fincham, and Tsang (2003) summarized the multiple definitions of forgive ness in this manner:

Despite the obvious differences among such definitions, they share an important feature - the assumption that forgiveness involves prosocial change regarding a transgressor on the part of the transgression recipient. Indeed, nearly every theorist appears to concur that when people forgive, their responses (i.e., thoughts, feelings, behavioral inclinations, or actual behaviors) toward a transgressor become more positive and/or less negative. (p. 540)

In fact, McCullough, Pargament, and Thoresen (2000) proposed that a "foundational and uncontroversial feature of forgiveness" is the "intraindividual prosocial change toward a transgressor" (McCullough et al., 2003, p. 540).

Two pieces of literature also provide definitions of interpersonal workplace forgiveness and organizational forgiveness that are geared specifically for discussions of forgiveness within workplace settings. Aquino, et al. (2003) explained that interpersonal workplace forgiveness is

a process whereby an employee who perceives himself or herself to have been the target of a morally injurious offense deliberately attempts to (a) overcome negative emotions (e.g., resentment, anger, hostility) toward his or her offender and (b) refrain from causing the offender harm even when he or she believes it is morally justifiable to do so. (p. 212)

Cameron and Caza (2002) defined organizational forgiveness as the capacity to foster collective abandonment of justified resentment, bitterness, and blame, and instead, it is the adoption of positive, forward-looking approaches in response to harm or damage. Forgiveness in organization requires a transformation, and an organization becomes virtuous to the extent to which it encourages, supports and facilitates such transformation. (p. 39) 
They also noted that the motive for individual or collective forgiveness cannot be instrumental: "Forgiveness in search of reward is not true forgiveness" (p. 39).

In sum, the definitions we have analyzed (some of which were presented here) all have common elements which provide the theoretical foundation for this paper. These elements were outlined effectively in Sells and Hargrave (1998). After an in-depth review of the theoretical and empirical literature on forgiveness, these researchers found that each forgiveness definition and theory contained the following six threads:

1. There is an injury or violation with subsequent emotional/physical pain.

2. The violation results in a broken/fragmented relationship between parties.

3. Perpetuation of injury is halted.

4. A cognitive process is pursued where the painful event or action is understood or reframed with a fuller context.

5. There is a release or letting go of justifiable emotion and retaliation related to the event.

6. There is a renegotiation of the relationship. (p. 28)

\section{Potential Impact of Forgiving}

Although the literature addresses the influence of forgiveness on many elements in one's life, this section will review the three most applicable to workplace and organizational performance: physical and mental health (well-being), job performance and productivity, and organizational issues.

\section{Physical and Mental Health}

The psychology and health literature provided a number of connections between forgiveness and physical health. Evidence suggests that chronic states of unforgiveness (i.e., anger, hostility, rumination, resentment, and fear) are linked to adverse physical health outcomes (Cameron \& Caza, 2002; McCullough, Bono, \& Root, 2007). Lamb (2005) discussed various studies that found forgiveness is related to higher individual ratings of physical health, decreased blood pressure, and fewer physician visits. According to Thoreson, Harris, \& Luskin (2000) forgiving responses "buffer ill-health by decreasing allostatic load and by promoting physiological and psychological healing" (as cited in Cameron \& Caza, 2002, p. 40). In addition, Harvard Women's Health Watch newsletter (2005) published an article on reasons to forgive. This article explained that unforgiveness creates anger, grudges, and feelings of injustice which creates a stress response and increases levels of stress hormones and increases in blood pressure and heart rate. When people retain these feelings for a prolonged period, the stress responses continue resulting in a "toll in their physical and emotional well-being" (p. 1). The authors report that forgiveness reduces stress, lowers heart disease risk, promotes healing from pain and illness, increases personal happiness, and improves interpersonal relationships.

Studies have also reported interesting findings regarding the connection between forgiveness and improved mental and emotional health (e.g., Anderson, 2007; Butler \& 
Mullis, 2001; Legaree et al., 2007; McCullough et al., 2007; Pettigrove, 2007). Lamb (2005) noted that the stress and hostility that comes from anger are known to be harmful to both one's physical and mental health. Scobie and Scobie (1998) stated that "forgiveness is reported to be a treatment which offers a means to overcome anger, resentment, the 'debilitating repletion of negative action' and to alleviate a persistent negative state" (p. 374). Forgiveness also lets individuals become "free" from the control of the offender. Zechmeister, Garcia, Romero, and Vas (2004) reported that forgiveness was associated with reduced anxiety and depression. Stone (2002) explained that when employees and managers have an in-depth understanding of the value of forgiveness, it actually provides opportunities to "use mistakes, failures, flaws and breakdowns of life as opportunities to awaken greater wisdom, compassion and capability in our coworkers and ourselves" (p. 279).

Three additional studies provide insight into the value of overall employee well-being. First, Little, Simmons, and Nelson (2007) examined positive behavior forgiveness and negative behavior revenge and their influence on an individual's health. They concluded that individuals who are more enthusiastic and actively engaged in positive enriching activities at work are often more healthy. Happy, healthy individuals are not typically those who are angry and hold grudges against others. Second, Bono and McCullough (2006) suggested a link between forgiveness and health because it may lead to "increased optimistic thinking and decreased hopelessness, increased self-efficacy, higher levels of perceived social and emotional support, and, for some, a greater sense of transcendent consciousness and communion with God" (p. 149), which ultimately promotes mental and physical health. Finally, Struthers, Dupuis, and Eaton (2005) found that interpersonal relationships in the workplace "are sometimes punctuated by a range of interpersonal offenses that can escalate into more serious damage to, and intractable conflict in, the relationship" (p. 306). Their research found that forgiveness is one process that may promote well-being at work.

\section{$\underline{\text { Job Performance and Productivity }}$}

A few researchers have reported the direct and indirect affects of forgiveness on job performance and productivity at work. Cameron, Bright, and Caza (2002) found that organizational forgiveness was "significantly associated with productivity after downsizing as well as lower voluntary employment turnover" (as cited in Cameron et al., 2002, p. 40). McCullough, Pargament, and Thoreson (2000) studied small organizations and found that forgiveness was associated with higher morale and satisfaction, greater social capital, trust, humanness, and caring relationships. This is particularly evident when an organization has experienced harm or unjust treatment as in cases of downsizing. Bottom et al. (2002) found that when individuals offer small substantive offers of penance, the likelihood of future cooperation was substantially enhanced. They noted these results are particularly pertinent due to the increased need for effective interpersonal relationships within and among organizations and employees in today's complex work environments. Further, forgiveness has been shown to motivate employees to "extend acts of conciliation and goodwill toward the offender and to overcome social estrangement" (Aquino et al., 2003, p. 213), which makes the working 
relationship between individuals more effective and productive. Forgiveness is actually a type of "problem-solving coping strategy in that it reconciles conflicting parties and salvages the social relationship for future interactions" (p. 213). Butler and Mullis (2001) conclude that when resentment and other negative feelings between coworkers exist, it is very difficult to maintain current levels of job performance let alone improve it.

\section{Organizational Issues}

Conflicts in the workplace may include situations such as coworkers having minor disagreements, departments at war with each other, hurtful rumors being spread, accurate or inaccurate performance appraisals, ethical and legal issues, employment decisions (e.g., hiring, firing, promotions), lack of support on initiatives or decisions, and more. Butler and Mullis (2001) noted that "personal offense stemming from norm violations at the interpersonal level constitutes the greatest number of workplace conflicts and frequently produces feelings of anger and resentment" (p. 259). The media writes and speaks of the increase in employee violence and acts of retaliation in workplaces throughout the country (Aquino et al., 2003). Many employees do not explore the various choices they have in choosing their reactions. In past years, companies have not addressed the issues of educating employees regarding coping mechanisms for a variety of situations. Today organizations are finding that training and development on a variety of new topics, competencies, and strategies is needed. Aquino et al. (2003) compared individual reactions to tragedies in the country with employee reactions to workplace violence and retaliation and stated that "workplace forgiveness has many of the same cognitive and emotional elements" as forgiveness does in other settings (p. 210).

The effects of interpersonal conflicts, resentment, hurt, and possible organizational injury varies based on transgression severity and an individual's forbearance when an incident occurs. When individuals forbear, endure, and/or control themselves when provoked (McCullough et al., 2003) the impact on the organization is lessened. Yet, "severe transgressions may be difficult to forbear because they can influence the transgression recipient's life more profoundly and pervasively" (p. 543). Transgression severity might also influence forgiveness. Severe transgressions tend to have more enduring consequences that may even be irreversible in both personal and work settings.

Unforgiving individuals within an organization and/or an unforgiving organizational culture in general can result in lower levels of performance at all levels. Davidhizar and Laurent (2000) found that when managers and leaders failed to forgive employees and superiors there was a lack of personal and team productivity that led "to aggressive and passive-aggressive behavior" on the part of the individual (p. 50). Stone (2002) wrote that the costs of not forgiving have "an enormous impact at each level of a system" ( $p$. 280), because employees separate themselves sometimes leading to ineffective job performance and/or leaving the organization. An organizational culture that does not promote forgiveness "will be engaged in negative and destructive politics" (p. 281) which will eventually decrease an organization's effectiveness. Hence, Stone believes 
that a forgiving culture improves retention, increases a sense of community, and "breeds authentic, courageous and open conversations" (p. 285).

\section{Examples of Workplace Forgiveness}

Episodes requiring forgiveness in the workplace can range from simple errors that may cause some difficulty or cost to the firm to major life-changing events such as downsizing and the accompanying layoffs or even national tragedies. They can impact an individual, a family, an entire company, or even the economy of a country.

Kurzynski (1998) discussed two episodes, one where forgiveness could have affected working relationships and the second where forgiveness was valuable. One employee was responsible for clipping and filing ads placed in industry trade journals by competitors. After several months, she took the initiative to write a report based on her files and send them to marketing management. The marketing department received her report well, but Carole's manager was angry about her "apparent lack of respect and disregard for the chain of command" (p. 78). Their working relationship became strained in the weeks that followed. The second example involved a medical technologist who was a recent graduate from college. She ran a batch of neo-natal blood tests without placing the guard on the centrifuge, and they were all destroyed (p. 78). Although the division head was very angry, the employee's supervisor supported her and was able to "see past the mistake of the novice technician, consider her performance to date, and recognize the potential of the person" (p. 83). Although the medical technologist was held accountable, the supervisor did not hold the incident against her. In fact, her supervisor suggested that "she devise a solution to the problem so that such an occurrence would not happen again" (p. 83). She wrote an effective procedure that ensured the equipment would always be used properly in the future.

Stone (2002) gave an example of working with a CEO and a VP of an organization to help them establish a better working relationship. The VP could not trust the CEO because of a situation ten years earlier. The CEO had not approved the VP's request for educational support, and the VP had resented and not trusted his boss for over a decade. After the CEO apologized, the VP forgave him and new "energy and enthusiasm entered their working relationship" (p. 281). According to Stone, "their new collaboration served as a working model and set an example that inspired the entire team to new levels of cooperation, collaboration, and open authentic communication" ( $p$. 281).

Family businesses often create problems that affect relationships. Hubler (2005) presented his Family Forgiveness Ritual ${ }^{\odot}$ (discussed elsewhere in this paper). He noted that

The assumptions, expectations, and role confusion that often plague family businesses can and do create major hurts ... It's not unusual in family businesses ... for siblings and parents and children not to be talking to each other or for various branches of the family to be excluding another branch. In some of the most dramatic cases, family members have sued each other. (p. 96) 
Hubler (2005) presented a case study in which a father and his oldest son were not speaking, even though they worked together every day. During the therapy, the son said, "I want to ask your forgiveness for taking so long to tell you that I was gay" (p. 101). The father responded, "I want to ask your forgiveness for how I handled hearing that you were gay" (p. 102). They participated in the ritual, and several years later, the mother reported "the business was doing very well and the family had never been better" (p. 102).

Cameron and Caza (2002) discussed Nelson Mandela as a striking example of an individual with "leadership virtue" and forgiveness. Although this example is of individual and national forgiveness, it has implications for the workplace. Mandela and the black population of South Africa had experienced decades of "unimaginable suffering and injustice" (p. 42). When the decision was made to hold free elections in South Africa, the world expected "revenge and retribution" as the "oppressed became the oppressors." The white minority government was to be replaced by black leadership. Mandela's example of "virtuous leadership led to an entirely different outcome" (p. 42). Cameron and Caza (2002) concluded, "The forgiveness exemplified by Mandela helped transform an entire nation" (p. 43). This remarkable example of how forgiveness could change a whole nation has implications for the value of forgiveness in a corporation, particularly when large problems such as downsizing can affect the lives of many employees and their families.

\section{Risks of Forgiveness}

In the psychological literature some concern is expressed about forgiving. McCullough (2000) stated that "forgiving might not universally be positively associated with health and well-being. It is possible that in certain interpersonal situations, people with a willingness to forgive might put their health and well-being at risk" (p. 51). He cited a study by Katz, Street, and Arias (1997) and indicated that "some research suggests that forgiveness may be a marker for relational disturbance, for example, in relationships characterized by physical abuse" (p. 51). While physical abuse may not be common in the workplace, other forms of abuse, such as sexual harassment, may be a problem that certainly cannot be accepted. Legaree and her associates (2007) suggested that victims focus on forgiveness of self, rather than on the perpetrator (p. 199). Care must be taken that forgiveness of such offenses is not seen as condoning them.

Although forgiveness should be perceived as an individual's strength, Kurzynski (1998) noted that problems can exist. "Unfortunately, the attitudes and beliefs people have with respect to forgiveness are often misconceived. Forgiveness is generally and often perceived as letting someone off the hook, forgetting, giving up or giving in, or being soft." However, he concludes that, "authentic forgiveness is [none] of these" (p. 80).

Cameron and Caza (2002) stated, "Despite misconceptions associating forgiveness with weakness or timidity, to truly forgive is an indication of remarkable streng th and discipline" (p. 38). They indicated that forgiveness does not imply forgetting, and that it takes time. They also said, "Forgiveness is distinct from trusting. Offenders need not be 
trusted just because they are forgiven. Abandoning negative emotions does not require that trust be re-established, even though a social relationship is renewed" (2002, pp. 3839).

Because the workplace often requires individuals to cooperate and even depend on one another, reconciliation may be important. However, this does not imply condoning the transgression. Aquino et al. (2003) stated, "Condoning an act is equivalent to denying the wrongness of an act. In contrast with forgiveness, it is essential that the injured party recognize that what the offender did was wrong...We can, in short, forgive the sinner but not condone the sin" (p. 213).

\section{Leadership and Management}

Stone (2002) argued that the primary purpose of leadership is to "create an environment of thriving, which allows people to grow, learn and contribute in a safe place where they feel they belong" (p. 278). He purports that "forgiveness is the most challenging and essential element of attaining a more nurturing and fulfilling climate at work" (p. 278). Yet many business leaders continue to believe that forgiveness is inappropriate to discuss in the workplace because it is considered an abstract philosophical or religious principle (Stone, 2002). However, it is becoming quite clear to successful leaders and managers that creating an enriching and creative environment requires teams and individuals to work together effectively (Little, Simmons, \& Nelson, 2007).

Researchers and practitioners have provided evidence regarding the role of managers and leaders in fostering a forgiving culture within their organizations. At times, it is this forgiveness leadership that allows organizations to move forward after difficult and sometimes devastating situations or occurrences. Cameron and Caza (2002) present two vital roles leaders can have in these circumstances. First, when leaders provide meaning and vision during these times, employees often respond positively. Forgiving does not mean that error is tolerated; it should actually "facilitate excellence and improvement rather than inhibiting it" (p. 44). Second, leaders can provide legitimacy and support. When leaders acknowledge and communicate the value of human development and welfare, a forgiving environment is enhanced. Leaders can exemplify, highlight, and celebrate virtuous actions, such as forgiveness, by initiating and supporting organizational structures, systems, and resources that are aligned with forgiveness and other important virtues (Cameron \& Caza, 2002). Kurzynski (1998) discussed the importance of forgiveness for managers when their employees make mistakes. He said that many managers expect perfect compliance, which is not a realistic expectation. The human element in business today, with its fast-paced, smaller staffed, and competitive environments, means that mistakes, disagreements, and problems are commonplace. Managers can be examples by accepting occasional mistakes and assisting employees so the mistakes are not repeated. Kurzynski argues that "forgiveness offers a way for the manager to deal with the negative and potentially destructive feelings that may result after a conflict between manager and employee in a way that can empower both (p. 79). He also spoke of the importance of forgiveness as an ethical response for managers. He believes that forgiveness is an ethical standard of 
behavior that requires managers to recognize and acknowledge the wrong. Forgiving requires the manager to "accept the responsibility and challenge in accepting others as human persons with and without their faults, and learning to live together without sustained anger and resentment" (p. 82). Kurzynaki defined ethical managers as forgiving individuals. Finally, Aquino, Tripp, and Bies (2006) conducted two empirical studies on revenge, forgiveness, and reconciliation. They concluded that creating a procedurally just climate seems to encourage forgiveness and reconciliation instead of revenge and avoidance. In doing this, not only will there be fewer offenses, but individuals will more likely utilize formal grievance processes in resolving their issues.

\section{Business Interventions}

The literature on workplace forgiveness interventions is limited, but numerous interventions are discussed in the psychological literature. In this section we present some of these studies and note their application to potential business interventions. Before doing this it is important to note that forgiveness interventions must acknowledge the implications and complexities of gender, culture, religion (Orr et al., 2005), class, and personal histories. In addition, various researchers and authors (e.g., Aquino, Tripp, \& Bies, 2006) extend a "call for action" for managerial efforts to "reengineer" their organizations "to include a procedurally just climate" (p. 666) that creates appropriate settings for forgiveness. The following list describes six intervention categories suggested in the literature:

1. Third-party interventions: Struthers et al. (2005) found that workers can be "encouraged to forgive such conflicts following third-party interventions developed to facilitate forgiveness" and that "by helping workers overcome the potentially debilitating reactions that can arise from conflict with their coworkers," (p. 306) forgiveness interventions can help repair damaged workplace relationships and assist in building more healthy workplaces.

2. Social interest interventions: Butler and Mullis (2001) argued that social interest increases forgiveness and that organizational development interventions may encourage the development of social interest, which would also increase a forgiving culture. Interventions such as intergroup team-building, third-party peacemaking, and survey feedback processes, which focus on social interest development in organizations, may "also promote forgiveness as a problemsolving strategy for workplace conflict” (p. 269).

3. Writing and journaling: Writing and journaling may be effective activities to aid in conflict management, conflict resolution, and interpersonal communication interventions within the workplace (Pettigrove, 2007). Professional counseling available through an Employee Assistance Program (EAP) may already be utilizing such methods ultimately as performance improving techniques. Landry, Rachal, Rachal, and Rosenthal (2005) studied the influence of writing and journaling about an interpersonal offense on a person's motivation to forgive. In their study with undergraduate students, they found that those who wrote about their conflict reported significant improvements in the manner they reflected on and experienced the interpersonal conflict. Participants who verbalized their thoughts and feelings also reported cognitive changes, improved understanding 
of the conflict, and improved coping skills (p. 3). Landry and her associates cited research that found the benefits of writing tended to persist and even grow stronger over time and that, although females benefit from writing, males benefit even more (p. 10).

4. Educational programs: Rodden (1997) discussed various dimensions of forgiveness in the framework of steps toward character education. Interestingly, character education is often an important component of workplace ethics training and development interventions. He recommends that steps toward forgiveness must be addressed in such educational programs. Hui and Ho (2004) found that forgiveness was a "viable classroom guidance program" (p. 477) that demonstrated student improvements in forgiveness, self-esteem, and hope. They did warn, however, that short programs (e.g., four weeks) are not enough time to demonstrate significant changes. They noted that group forgiveness interventions were effective in helping some participants forgive specific individuals but not others in general. Denton and Martin (1998) argued that "education about forgiveness is essential in emphasizing the power of individual choice, the personalized aspects of its process, and its real benefits" (p. 289). Education can empower employees by providing knowledge of what is happening as well as promoting awareness of the possible biases and assumptions they may have which may hinder the forgiveness process.

5. Forgiveness programs: Hubler (2005) reported on his use of a Family Forgiveness Ritual(C, which he found to be effective with problems incurred in family-owned businesses. This intervention used individual counseling and the inclusion of a member of the clergy selected by the family. While this type of intervention may be useful for a small, close-knit group such as a family, it may not be as helpful in a larger organization. A time may arise when an individual should be referred to a therapist for help with anger management, which might lead to forgiveness therapy. Some type of group forgiveness therapy may be appropriate for situations such as a major downsizing or a hostile takeover. It would appear that training of leaders in modeling forgiveness as well as general training in conflict resolution might be more effective.

6. Empathy-building and communication interventions: McCullough et al. (2003) found that individuals tended to have more forbearance if the transgression was not severe, if they had empathy with the transgressor, and if they did not have strong attribution of responsibility towards the transgressor. While the severity of the transgression may be beyond the control of management, it would appear that building strong empathy among employees would be helpful in encouraging forbearance and forgiveness. Communication might also mitigate the impact of responsibility attribution, although it appears to be important not to make excuses for genuine transgressions. Pettigrove (2007) includes a caution, however, that understanding does not always lead to forgiving as it may be perceived as excusing and justifying. Bottom et al. (2002) noted that "accepting blame may create better prospects for future cooperation in short interactions. By admitting a mistake, a person acknowledges their fallibility and may even generate increased attraction" (p. 510). 
Not all of the interventions presented in this paper can be offered directly by an HRD or human resources department. Yet, if leaders, managers, and HR/HRD professionals are educated and aware of the counseling options, they can provide employees with appropriate suggestions and recommendations for further assistance. For example, many companies offer employee assistance programs that provide professional counseling for various emotional issues and stressful situations. The use of EAPs by organizations has increased in past years as more businesses are finding that physically and mentally healthier employees are more productive. These interventions have shown to lead to improved workplace performance at all levels.

\section{Proposed Framework}

Based upon the reviewed literature, we propose the following integrated perspective of forgiveness: forgiveness is a psychological act, a communicative act, and a social act (see Figure 1). At the individual (psychological) level it involves letting go of offense even if being offended is justified and the hurt is sustained. At the dyadic (communication) level, it involves letting the offending party know that the offense has been removed or erased. At the organizational/cultural (social) level, it means that the relationship and associations are in balance and functioning effectively. A set of elements and questions for each of these levels of forgiveness provides the framework for scholars and practitioners to analyze and understand this phenomenon at a more comprehensive level.

\section{Individual Level (Psychology)}

1. Perception: Has an offense been seen, heard or felt? People perceive the intent to offend differently. For example, the literature suggests that performance feedback is often perceived as offensive. Yet others value feedback from trusted individuals as a gift. People with a long history of violence or abuse may also perceive offense differently.

2. Justification: Are the feelings of hurt justified? Some individuals "own" the hurt, saying the offender was justified, while others personalize and amplify their feelings.

3. Sustained: Is the feeling of hurt sustained? For some individuals an offense is felt over time, while others can "let go" quickly. Several studies suggest males accumulate fewer but more significant conflicts. Sustained conflict comes when the probability or anticipation of reoccurrence is felt.

\section{Dyadic Level (Communication)}

1. Identity or action: Is the offence against your identity (who you are) or your action (what you did)? What is the difference in perception between the offending party and the offender?

2. Retaliation, defense, or acceptance: People hold assorted learned strategies for dealing with offense or hurt. Is the offended party justifying retaliation (verbal or 
behavioral), being defensive (keeping additional hurt from happening), or accepting (playing the victim or "absorbing" the hurt)?

3. History: Is there a history? Do the offender and the offended have a sustained relationship and a history of resolution?

Figure 1: An Integrated Model of Forgiveness

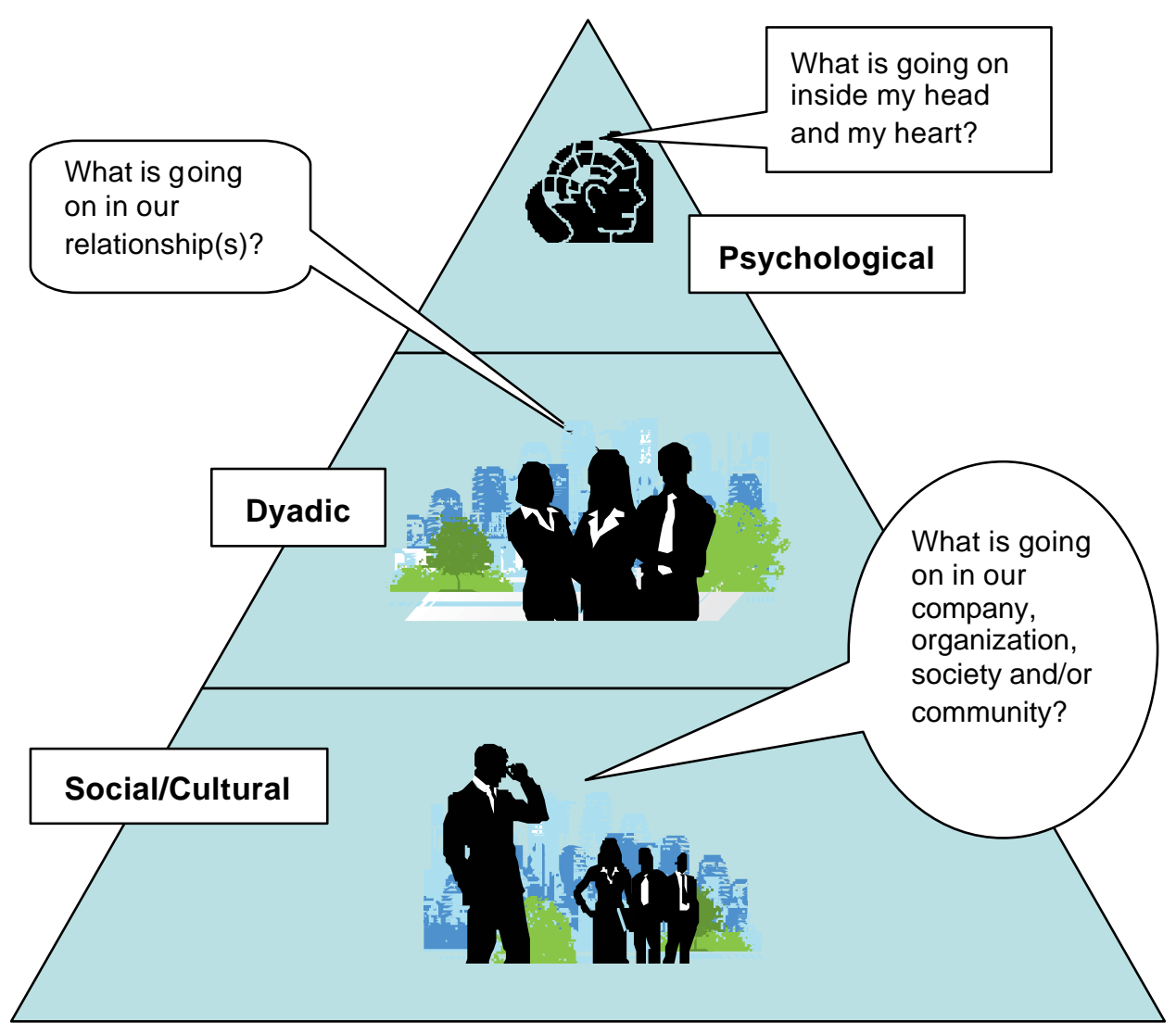

\section{Organizational/Culture Level (Social)}

1. Moral Values: Is forgiveness valued beyond just the functional aspect of a relationship?

2. Trust: Can trust be restored between the offender and the offended, and can hurt be forgotten?

3. Harmony: Can balance and harmony be restored so that the parties can work productively together?

This simple model highlights the difficulty of dealing with issues of forgiveness in an organizational setting. We note that the literature on forgiveness can be loosely categorized into psychological, communicative and social categories. As an offense 
perceived creates a psychological need for forgiveness, forgiveness cannot be consummated unless it is communicated to the offending party. When forgiveness goes public in a communicative act, human subjectivity reigns. The legitimacy of the offense and the perception of harm come into dispute. At the social/cultural level, these issues often play out in social discourse where the offenses are voiced and contexted in what can become a revictimization of the wronged or a villanization of the offender, further exacerbating the hurt. Scholars and HR professionals alike clearly need to conceptualize the complexity of this subject while providing practical ways to reduce hurt through forgiveness.

\section{Conclusion and Recommendations}

A primary focus of management and HRD is to improve individual, team and organizational performance. This is the first manuscript to suggest that HRD researchers and practitioners should consider the influence of individual and workplace forgiveness on the performance and productivity of employees and organizations, and one of the first to suggest that management researchers and practitioners to do the same. The literature reviewed in this paper provides a solid foundation for management and HRD professionals to consider the potential impact of forgiving on physical and mental health, job performance and productivity, and organizational issues. It makes a case for the role of leaders and managers in creating an environment (which includes forgiveness) that allows for effective learning, development, and contribution of all employees. This paper also reviews the literature on various change interventions focused on forgiveness that can lead to performance improvements. Finally, it presents a new forgiveness framework for scholars and practitioners to use in analyzing and understanding this phenomenon.

Workplace forgiveness is a new area of inquiry, and research is needed to understand this and other related constructs (e.g., revenge). For example, does our ability to forgive positively contribute to our productivity? Do organizations with a capacity to forgive employees have a sustained competitive advantage? Can forgiveness interventions be effective? Can the capacity to forgive be expanded through training? Is forgiveness just a moral value, or does it have practical value? Although these and other questions require further exploration, we believe that these explorations should not be isolated. Further exploration would benefit from incorporating the different levels (individual, dyadic, and organization/culture) and types (psychological, communicative, and social) of forgiveness we have presented in this model.

\section{References}

Aquino, K., Grover, S. L., Goldman, B., \& Folger, R. (2003). When push doesn't come to shove: Interpersonal forgiveness in workplace relationships. Journal of Management Inquiry, 12(3), 209-216.

Aquino, K., Tripp, M. T., \& Bies, R. J. (2006). Getting even or moving on? Power, procedural justice, and types of offense as predictors of revenge, forgiveness, 
reconciliation, and avoidance in organizations. Journal of Applied Psychology, 91 (3), 653-668.

Anderson, J. (2007). Forgiveness-a relational process: Research and reflections. European Journal of Psychotherapy and Counseling, 9(1), 63-76.

Bono, G., \& McCullough, M. E. (2006). Positive responses to benefit and harm: Bringing forgiveness and gratitude into cognitive psychotherapy. Journal of Cognitive Psychotherapy: An International Quarterly, 20(2), 147-158.

Bottom, W. P., Gibson, K., Daniels, S. E., \& Murnighan, J. K. (2002). When talk is not cheap: Substantive penance and expressions of intent in rebuilding cooperation. Organization Science, 13(5), 497-513.

Butler, D. S., \& Mullis F. (2001). Forgiveness: A conflict resolution strategy in the workplace. The Journal of Individual Psychology, 57(3), 259-272.

Cameron, K., \& Caza, A. (2002). Organizational and leadership virtues and the role of forgiveness. Journal of Leadership \& Organizational Studies, 9(1), 33-48.

Davidhizar, R. E., \& Laurent, C. R. (2000). The art of forgiveness. Hospital Materiel Management Quarterly, 21(3), 48-53.

Denton, R. T., \& Martin, M. W. (1998). Defining forgiveness: An empirical exploration of process and role. The American Journal of Family Therapy, 26, 281-292.

Enright, R. D. \& Coyle, C. T. (1998). Researching the process model of forgiveness within psychological interventions. In E. L. Worthington Jr. (Ed.), Dimensions of forgiveness: Psychological research and theological perspectives (pp. 139-161). Philadelphia: Templeton Foundation Press.

Harvard Medical School. (2005). Five for 2005: Five reasons to forgive. Harvard Women's Health Watch, 12(5), 1-3.

Hubler, T. M. (2005). Forgiveness as an intervention in family-owned business: A new beginning. Family Business Review, 18(2), 95-103.

Hui, E. K. P., \& Ho, D. K. Y. (2004). Forgive ness in the context of developmental guidance: Implementation and evaluation. British Journal of Guidance \& Counseling, 32(4), 477-492.

Kurzynski, M. J. (1998). The virtue of forgiveness as a human resource management strategy. Journal of Business Ethics, 17(1), 77-85.

Lamb, S. (2005). Forgiveness therapy: The context and conflict. Journal of Theoretical and Philosophical Psychology, 25(1), 61-80. 
Landry, D. F., Rachal, K. C., Rachal, W. S., \& Rosenthal, G. T. (2005). Expressive disclosure following an interpersonal conflict: Can merely writing about an interpersonal offense motivate forgiveness? Counseling and Clinical Psychology Journal, 2(1), 2-14.

Legaree, T.-A., Turner, J., \& Lollis, S. (2007). Forgiveness and therapy: A critical review of conceptualizations, practices, and values found in the literature. Journal of Marital and Family Therapy, 33(2), 192-213.

Little, L. M., Simmons, B. L., \& Nelson, D. L. (2007). Health among leadership: Positive and negative affect, engagement and burnout, forgiveness and revenge. The Journal of Management Studies, 44(2), 243-260.

McCullough, M. E. (2000). Forgiveness as a human strength: Theory, measurement, and links to well-being. Journal of Social and Clinical Psychology, 19(1), 43-55.

McCullough, M. E., Bono, G., \& Root, L. M. (2007). Rumination, emotion, and forgiveness: Three longitudinal studies, Journal of Personality and Social Psychology, 92(3), 490-505.

McCullough, M. E., Fincham, F. D., \& Tsang, J. (2003). Forgiveness, forbearance, and time: The temporal unfolding of transgression-related interpersonal motivations. Journal of Personality and Social Psychology, 84(3), 540-557.

McCullough, M. E., Pargament, K. I., \& Thoreson, C. (2000). Forgiveness: Theory, research, and practice. New York: Guilford.

Orr, R. R., Sprague, A. M., Goertzen, L. R., Cornock, B. L., \& Taylor, D. P. (2005). Forgiveness in a counseling context: Definition and process. Guidance \& Counseling, 20(2), 71-77.

Pettigrove, G. (2007). Understanding, excusing, forgiving, Philosophy \& Phenomenological Research, 74(1), 156-175.

Rodden, J. (1997). Dimensions of forgiveness: An untried step toward character education. Vital Speeches of the Day, 63(23), 712-718.

Scobie, E. D., \& Scobie, G. E. W. (1998). Damaging events: The perceived need for forgiveness. Journal for the Theory of Social Behavior, 28(4), 373-401.

Sells, J. N., \& Hargrave, T. D. (1998). Forgiveness: A review of the theoretical and empirical literature. Journal of Family Therapy, 20, 21-36.

Smith, H. (1991). The worlds religions. San Francisco: Harper Collins. 
Stone, M. (2002). Forgiveness in the workplace. Industrial and Commercial Training, 34(6/7), 278-286.

Struthers, C. W., Dupuis, R., \& Eaton, J. (2005). Promoting forgiveness among coworkers following a workplace transgression: The effects of social motivation training. Canadian Journal of Behavioral Science, 37(4), 299-308.

Zechmeister, J. S., Garcia, S. Romero, C., \& Vas, S. N. (2004). Don't apologize unless you mean it: A laboratory investigation of forgiveness and retaliation. Journal of Social and Clinical Psychology, 23(4), 532-564. 